\section{What do we know about covid vaccines and preventing transmission?}

stokel@gmail.com

Cite this as: BMJ 2022;376:0298

http://dx.doi.org/10.1136/bmj.0298

Published: 04 February 2022

Vaccines that work against SARS-CoV-2 have helped change the course of the pandemic by reducing illness and hospital admissions. But Chris Stokel-Walker asks what we know about their impact on preventing transmission.

\section{Chris Stokel-Walker freelance journalist}

The range of vaccines developed in record time by pharmaceutical companies and research laboratories have helped quell the worst effects of SARS-CoV-2. But much of the focus of research has been on effectiveness in preventing infection, illness, and hospital admission. What is less well measured is the impact of vaccination on preventing onward transmission.

\section{What evidence do we have that covid-19 vaccines prevent transmission?}

Most papers to date (notably, many are preprints and have yet to be peer reviewed) indicate vaccines are holding up against admission to hospital and mortality, says Linda Bauld, professor of public health at the University of Edinburgh, "but not so much against transmission."

The first weekly covid-19 vaccine surveillance report for $2022^{1}$ from the UK Health Security Agency (UKHSA) was more positive than Bauld's assessment-but didn't say outright that covid-19 vaccines prevent transmission. "Several studies have provided evidence that vaccines are effective at preventing infection," it states, "Uninfected people cannot transmit; therefore, the vaccines are also effective at preventing transmission."

A study ${ }^{2}$ of covid-19 transmission within English households using data gathered in early 2021 found that even a single dose of a covid-19 vaccine reduced the likelihood of household transmission by 40-50\%. This was supported by a study of household transmission among Scottish healthcare workers conducted between December 2020 and March 2021. ${ }^{3}$ Both studies analysed the impact of vaccination on transmission of the $\alpha$ variant of SARS-CoV-2, which was dominant at the time.

A subsequent study, ${ }^{4}$ conducted later in the course of the pandemic when the delta variant was dominant, showed vaccines had a less pronounced effect on denting onward transmission, but were still effective.

\section{How could vaccines help reduce transmission?}

Vaccines aren't preventing onward transmission by reducing the viral load-or amount of SARS-CoV-2-in your body. "Most studies show if you got an infection after vaccination, compared with someone who got an infection without a vaccine, you were pretty much shedding roughly the same amount of virus," says Paul Hunter, professor in medicine at the University of East Anglia. One study, ${ }^{5}$ sponsored by the US Centers for Disease Control and Prevention (CDC), found "no difference in infectious virus titer between groups" who had been vaccinated and had not.

Instead, it's the principle that the UKHSA identified above: if you don't get infected in the first place thanks to a vaccine, you can't spread it. Once you're infected, you still can-although what we know about the window when you're most likely to transmit the virus to others has improved.

\section{Does the omicron variant make a difference?}

Few studies have looked at the omicron variant, although a report published in January 2022 by the European Centre for Disease Prevention and Control cited a small Danish household study: "People who have completed the primary series of vaccination experienced secondary attack rates (SARs) of 32\% in households with omicron and $19 \%$ in households with delta. For people who received a booster, omicron was associated with a SAR of $25 \%$, while the corresponding estimate for delta was only $11 \%$. There was an increased transmission for unvaccinated people, and a reduced transmission for booster vaccinated people, compared with fully vaccinated people," summarised the report. ${ }^{7}$

Preliminary data from Japan's National Institute of Infectious Diseases found that patients infected with omicron shed viral particles for longer compared with those infected with other variants. The amount of viral RNA in patients with omicron was highest three to six days after diagnosis or symptom onset. This appears to be two or three days later than other variants. ${ }^{8}$ Hunter said the new data "muddy the waters" on the matter.

Vaccine effectiveness against infection, hospital admission, and mortality have all taken a hit when pitted against the omicron variant, and it seems only logical that the impact against transmission would likewise drop.

"The main point of vaccines is not to do with preventing transmission," says Anika Singanayagam, academic clinical lecturer in adult infectious disease at Imperial College London. "The main reasons for vaccines for covid-19 is to prevent illness and death." Therefore, we shouldn't be too disappointed that it's still possible to pass on the virus while vaccinated, she says, "Damping down on transmission is not a particularly easy thing with omicron."

\section{What impact does that have on policymaking?}

The fact that vaccines are good at preventing serious infection, but less good at preventing transmission 
makes policymaking difficult. The UK has changed its rules ${ }^{9}$ on the amount of time those who test positive for covid-19 must spend in self-isolation, first from 10 days to seven, then to five, provided they test negative on a lateral flow test. That decision follows the US, which cut the self-isolation period to five days in late December ${ }^{10}$ because "the majority of SARS-CoV-2 transmission occurs early in the course of illness."

"They're recognising that vaccines aren't preventing transmission, and you've got too many people having to isolate," says Bauld.

"Policymakers have decided that the game's up on transmission, but that you need a different approach."

Decision makers have a difficult decision, says Singanayagam: they want to enable life to continue as normally as possible-which may mean vaccinated people getting infected with covid because of community or household transmission-while also carefully monitoring that vaccine effectiveness to lower the risk of hospital admission, severe illness, and death is not dented.

\section{Could future vaccines be more effective against onward transmission?}

Again, first generation covid vaccines were evaluated against reducing hospital admissions and death in the challenging first year of the pandemic. They wouldn't have been expected to generate sterilising immunity and block transmission. But, says

Singanayagam, now that we have a suite of vaccines using different approaches, there is some opportunity to think about future jabs for different situations.

"There are avenues to think about the development of vaccines that can have more of an effect on transmission," she says. Those are usually vaccines delivered more locally, such as directly through the respiratory tract, which could tackle the source of major transmission, rather than the lungs, which is where the first generation of vaccines was targeted in order to prevent severe infection. "That's probably the way things will move in the future."

1 UK Health Security Agency. Covid-19 vaccine surveillance report: week 1. 6 January 2022. https://assets.publishing.service.gov.uk/government/uploads/system/uploads/attachment_data/file/1045329/Vaccine_surveillance_report_week_1_2022.pdf

2 Harris RJ, Hall JA, Zaidi A, Andrews NJ, Dunbar JK, Dabrera G. Effect of vaccination on household transmission of SARS-CoV-2 in England. N Engl J Med 2021;385:759-60. doi: 10.1056/NEJMc2107717 pmid: 34161702

3 Shah ASV, Gribben C, Bishop J, etal. Effect of vaccination on transmission of covid-19: SARS-Cov-2 N Engl J Med 2021;385:1718-20. doi: 10.1056/NEJMc2106757 pmid: 34496200

4 Eyre DW, Taylor D, Purver M, etal. The impact of SARS-CoV-2 vaccination on alpha and delta variant transmission.Medrxiv2021.09.28.21264260 [Preprint]. doi: 10.1101/2021.09.28.21264260

5 Riemersma KK, Grogan BE, Kita-Yarbro A, etal. Shedding of infection SARS-CoV-2 despite vaccination.Medrxiv 2021.07.31.21261387 [Preprint]. doi: 10.1101/2021.07.31.21261387

6 Lyngse FP, Mortensen LH, Denwood MJ, etal. SARS-CoV-2 omicron VOC transmission in Danish households.Medrxiv 2021.12.27.21268278 [Preprint]. doi: 10.1101/2021.12.27.21268278

7 European Centre for Disease Prevention and Control. Assessment of the further spread and potential impact of the SARS-CoV-2 omicron variant of concern in the EU/EEA, 19th update. 27 January 2022. www.ecdc.europa.eu/en/publications-data/covid-19-omicron-risk-assessmentfurther-emergence-and-potential-impact

8 Torjesen I. Covid-19: Peak of viral shedding is later with omicron variant, Japanese data suggest. BMJ 2022;376:089. doi: 10.1136/bmj.089 pmid: 35027360

9 Mayor S. Covid-19: Warning over transmission risk as self-isolation is cut to five days in England. BMJ 2022;376:0111. doi: 10.1136/bmj.o111 pmid: 35031534

10 Centers for Disease Control and Prevention. CDC updates and shortens recommended isolation and quarantine period for general population. 27 December 2021. www.cdc.gov/media/releases/2021/s1227-isolation-quarantine-guidance.html 